\title{
"Meglio ancora delle sue opere, che nessuno più legge":" note per la riscoperta di Maddalena Campiglia (1553-1595)
}

\author{
Anna Lisa Somma \\ annalisasomma@gmail.com
}

\begin{abstract}
Sebbene lodata in vita per l'onestà e la saggezza, Maddalena Campiglia (Vicenza 1553-1595) è stata a lungo trascurata dagli studiosi. La sua produzione non è copiosa e consta di tre opere principali. La prima, il Discorso sopra l'Annonciatione della Beata Vergine, \& la Incarnatione del S.N. Giesu Christo (1585), parrebbe a prima vista un comune libro devozionale, mentre contiene interessanti critiche all'istituzione matrimoniale (d'altronde, la Campiglia stessa era separata dal marito). Flori (1588) è invece una delle rare favole pastorali composte da una donna nell'Italia rinascimentale. La scrittrice sembra qui accettare il classico immaginario pastorale e le sue convenzioni, per poi infrangere le aspettative di gender e di genre, introducendo in una tradizione antica una serie di elementi trasgressivi. Anche il proseguimento ideale del lavoro, Calisa (1589), un "epithalamium interruptum" (ULTSCH (I) 2005), si rivela eterodosso: la protagonista critica l'istituto nuziale e, grazie alla sua eloquenza, dimostra la liceità dell'amore saffico.
\end{abstract}

Parole chiave: Maddalena Campiglia. Letteratura italiana rinascimentale. Proto-femminismo. Maternità letteraria. Scritture femminili.

\footnotetext{
${ }^{1}$ Il giudizio completo di Rumor recita: "Questo largo tributo di lode, dato dal Tasso alla poetessa Vicentina, meglio ancora delle sue opere, che nessuno più legge, ne ha assicurato la fama, e reso popolare il nome quasi quanto quello di Gaspara Stampa e di Vittoria Colonna". Il parere - tratto da Rumor (1897, pp. 9-10) - è riportato in Chemello (2003, p. 65).
} 
"Meglio ancora delle sue opere, che nessuno più legge": note per la riscoperta di Maddalena

Campiglia (1553-1595)

Anna Lisa Somma

\begin{abstract}
Although praised during her life for her honesty and wisdom, Maddalena Campiglia (Vicenza 1553-1595) has been neglected for long time and her books have been overlooked by scholars. Her production is not voluminous and consists of three main works. The first one, the Discorso sopra l'Annonciatione della Beata Vergine, \& la Incarnatione del S.N. Giesu Christo (1585), appears like a common devotional book, but it contains interesting criticism of marriage (Campiglia separated from her husband). Flori (1588) is one of the rare pastoral dramas written by Renaissance female writers in Italy. Campiglia seems to accept the classical pastoral imagery and its conventions, but she actually reworks the genres and breaks gendered expectations, introducing many transgressive elements into an ancient tradition. Also the sequel of the work, Calisa (1589), an "epithalamium interruptum" (ULTSCH 2005), is heterodox. There Flori criticizes the nuptial institution and thanks to her eloquence demonstrates the lawfulness of Saffic love.
\end{abstract}

Keywords: Maddalena Campiglia. Italian Renaissance Literature. Protofeminism. Literary maternity. Women's writing.

\title{
1. Introduzione
}

Il viso precocemente invecchiato, il corpo femmineo nascosto sotto una veste scura e la mano destra posata su un fascio di fogli, i suoi scritti: così ci appare nel ritratto eseguito da Alessandro Maganza la berica Maddalena Campiglia (1553-1595), autrice poco nota alla critica italiana, ma capace di attrarre su di sé l'attenzione degli studiosi stranieri (in particolare angloamericani). Essi hanno sottolineato l'originale valore delle sue scelte stilistiche e delle sue riflessioni anticonformistiche, non di rado in relazione al coevo panorama letterario muliebre della Repubblica di Venezia, in cui videro la luce 
"Meglio ancora delle sue opere, che nessuno più legge": note per la riscoperta di Maddalena

Campiglia (1553-1595)

Anna Lisa Somma

opere dall'ispirazione proto-femminista quali Il merito delle donne (1600) di Modesta da Pozzo, La nobiltà et l'eccellenza delle donne (1600) di Lucrezia Marinella e l'Inferno Monacale (prima metà del XVII sec.) di Arcangela Tarabotti. $^{2}$

\section{La vita}

Pia, erudita, stimata dagli intellettuali: questo è quanto trapela dalle opere di Maddalena e dalle testimonianze letterarie a lei dedicate dai contemporanei. Proveniente da una rinomata famiglia, sin dalla nascita (avvenuta a Vicenza il 13 aprile 1553) la scrittrice esperì una condizione sociale alquanto eccentrica per l'epoca: i suoi genitori, i nobili Carlo Campiglia e Polissena Verlato - vedovi, ciascuno con propria prole - si sposarono solamente nel 1565, quando la bambina aveva dodici anni e, con buone probabilità, il rapporto non fu accettato pacificamente dai parenti di entrambi.

Possediamo scarse informazioni sull'educazione di Maddalena, che dovette essere in linea col tempo. Sebastiano Rumor ci informa che la donna

\footnotetext{
coltivò [...] con singolare predilezione le lettere e la musica, e nelle une e nell'altra riuscì valente. Negli ultimi anni di sua vita si doleva di aver abbandonato, benché da poco tempo, "le viole e i liuti e gli arpicordi" da lei "stimati e cari avuti", coi quali faceva la sua parte "se non lodatamente a pieno, almen sicura”. (RUMOR 1897, p. 10)
}

Forse spinta dalla famiglia, nel gennaio 1576 sposò il nobile Dionisio Colzè, un personaggio del quale conosciamo ben poco, ma la cui importanza nella vita di Maddalena Campiglia fu estremamente significativa, seppure, a quanto ci è dato sapere, in chiave negativa. Se al momento vi sono forti

\footnotetext{
2 Riguardo la nascita del cosiddetto proto-femminismo rinascimentale cfr. Jordan (1990) e Ross (2009).
} 
"Meglio ancora delle sue opere, che nessuno più legge": note per la riscoperta di Maddalena

Campiglia (1553-1595)

Anna Lisa Somma

incertezze sullo svolgimento della consueta convivenza tra i coniugi, è ormai appurato che già agli anni $1581-1583$ si può far risalire la rottura tra moglie e marito, sulle cui cause allo stato attuale non vi sono certezze; per Morsolin essa sarebbe da imputare a un "[...] amore soverchio e diciamo pure sregolato [di Maddalena] a non si sa quale amica morta nel fiore degli anni e della bellezza” (MORSOLIN 1882). ${ }^{3}$ In ogni caso, è forse lecito ipotizzare - anche sulla scorta dei legati di Carlo Campiglia - un'unione matrimoniale dal principio basata più su interessi economici (soprattutto da parte dei familiari di Maddalena) che su una reale affinità. A questo proposito è senza dubbio pregno di significato il fatto che, già nell'aprile 1576, la fanciulla chiedesse con "strana istanza" che negli incartamenti relativi alla dote fossero aggiunti tutti gli oggetti d'oro e d'argento da lei recati in dote, in vista di "casus restituende dimidie dotis propter deficentiam filiorum ex dicto matrimonio" (MANTESE 1967, p. 105).

Il testamento dettato da Dionisio Colzè il 12 settembre 1577 dona nuovi, utili dettagli riguardo la natura (finanziaria e non solo) del rapporto. Da esso emerge "il rifiuto netto e totale dei fratelli di Maddalena di correspondere la dote pattuita" (GHERARDI 2009, p. 17), la quale gli sarebbe stata poi versata - e solo per metà - addirittura nel 1609, dopo la morte dell'ex coniuge e lunghe contese giudiziarie; i cognati, inoltre, avrebbero in qualche modo offeso Dionisio "in honore et vita [...] presertim quod ipse noluit consentire nec acceptare domi quamdam meretricem" (GHERARDI 2009, pp. 17-18). Chi e cosa spinse l'uomo a una reazione verbale così aggressiva? E può la meretrix identificarsi con la scrittrice?

\footnotetext{
${ }^{3}$ Questa idea potrebbe forse esser stata (almeno in parte) condizionata dai due amori lesbici messi in scena dall'autrice nelle sue opere pastorali.
} 
"Meglio ancora delle sue opere, che nessuno più legge": note per la riscoperta di Maddalena

Campiglia (1553-1595)

Anna Lisa Somma

Come accennato sopra, la fine della relazione dovette aver luogo tra il giugno 1581 e il maggio 1583, mese in cui la donna risultava già essere tornata nella casa paterna. Proprio nel 1583 nominò suo procuratore Bernardino Marana per dirimere le liti di pertinenza del foro civile e religiosi riguardanti il suo ex marito; l'operazione, però, dovette rivelarsi più complessa del previsto se persino nella sua produzione letteraria, e a più riprese, Maddalena lamentò il peso dell'“antico gioco indegno" matrimoniale (CAMPIGLIA 1996, v. 216) e addirittura ne richiese lo scioglimento. ${ }^{4}$

Certo le relazioni tra i coniugi non furono delle più pacifiche, dal momento che, nel testamento (1593), Maddalena lasciò scritto con tono sprezzante:

Et se per caso, in tempo alcuno, un certo asserto Dioniso Colzè pretendesse o movesse lite alcuna sopra i beni di detta Signora Testatrice et contro li suoi heredi, lei dice et protesta che lui non pole haver né conseguir cosa alcuna perché in coscientia, alla ragion d'Iddio, non è stato mai suo marito né pol conseguire né deve cosa cosa. (MORSOLIN 1882, pp. 66-72, cit. in MANTESE 1967, p. 98, n. 23)

Il matrimonio - a quanto si delinea dalle parole di Maddalena sembrerebbe dunque non esser stato consumato, sebbene le ragioni di ciò ci sfuggano, e siamo all'oscuro di eventuali azioni (legali e non solo) del marito in concomitanza con la conclusione del legame.

Una volta separatasi, Maddalena venne a trovarsi al di fuori delle usuali classificazioni sociali del tempo: né donna da maritare, né maritata e tanto

\footnotetext{
${ }^{4}$ Inoltre, come rileva Adriana Chemello, in due sonetti encomiastici pubblicati insieme al Trofeo della Croce di Cesare Calderari, la Campiglia si indirizzò all'autore stesso e al vescovo di Vicenza, Michele Priuli, affinché intervenissero per liberarla dall'increscioso legame nuziale: "L'Agnello arso d'amor miro e non ardo?/ Inceneriamo o gran MICHEL; ma i nodi/ gravi miei tu disciogli, anzi, ch'io mora." e "Conte di te le lodi. Ah, quando quando/ io far potrò, benigno almo Pastore,/ sciolta da giogo, on'io ne pero amaro" (CALDERARI 1588, cit. in CHEMELLO 2003, pp. 89-90).
} 
"Meglio ancora delle sue opere, che nessuno più legge": note per la riscoperta di Maddalena

Campiglia (1553-1595)

Anna Lisa Somma

meno vedova. In questa rigida tassonomia, le nubili occupavano una posizione anomala e poco lusinghiera (naturalmente le religiose costituivano un caso a sé stante). ${ }^{5}$ Crediamo sia anche per queste ragioni storico-sociali che si è a lungo dato credito allipotesi della monacazione della Campiglia, smentita solo nella seconda metà del Novecento (MANTESE 1967): imbattersi in una simile gentildonna, colta, autonoma e, soprattutto, incurante dei giudizi altrui non doveva esser frequente nella Vicenza controriformistica. ${ }^{6}$

È tuttora ancora viva, invece, la congettura secondo la quale Maddalena possa aver aderito al movimento devozionale secolare delle dimesse - di cui indosserebbe l'abito nel ritratto descritto in apertura del nostro lavoro -, sorto a Vicenza nel 1579 (fra le maggiori sostenitrici della sua nascita vi è da ricordare anche Maddalena Pigafetta, zia materna di Dionisio Colzè), libero dalla giurisdizione ecclesiastica e non soggetto alla rigida disciplina imposta dal Concilio di Trento, specialmente in tema di clausura. ${ }^{7}$

Occorre non sottovalutare questi dati, reputandoli dotte e futili curiosità: non solo, infatti, le possibili affiliazioni religiose di Maddalena costituiscono fonte di dibattito dal XVIII secolo, ma - soprattutto - da esse è più volte dipesa l'esegesi delle opere della vicentina, specie tenendo conto di una presunta vocazione religiosa che nel corpus troverebbe la sua consacrazione.

Ritornando alla biografia della donna, in seguito alla separazione, Campiglia iniziò un'intensa fase scrittoria, concentrata soprattutto nel lustro

\footnotetext{
${ }^{5}$ Sulle coeve condizioni della donna cfr. almeno Duby e Perrot (2009).

${ }^{6}$ Nota Diana Sartori: “[q]uella falsa pista della monacazione è però indicativa dell'esistenza di una difficoltà a inquadrare la figura di Maddalena riportandola ad una delle tradizionali categorie di iscrizione delle donne in base alla condizione civile: da maritare, maritata, vedova." (SARTORI 1994, p. 59).

7 Sulla clausura cfr. per esempio Zarri (2000).
} 
"Meglio ancora delle sue opere, che nessuno più legge": note per la riscoperta di Maddalena

Campiglia (1553-1595)

Anna Lisa Somma

1585-1590; dapprima la scrittrice pubblicò l'opera devozionale Discorso sopra l'Annonciatione della Beata vergine e la Incarnatione del S. N. Giesù Christo (1585), cui seguirono la favola pastorale Flori (1588) e l'egloga Calisa (1589). Ai testi più noti possiamo aggiungere alcuni sonetti e madrigali editi in diverse raccolte (come quelle di Calderari, Baldi e Grillo), e abbiamo notizia anche di una Vita di santa Barbara perduta. Maddalena Campiglia compose inoltre trentasei argomenti in ottave che fungono da prefazione ai canti della seconda edizione del Fidamante (1591), nonché un'epistola dedicatoria per Gl'inganni (1592), entrambi di Curzio Gonzaga.

Nel medesimo periodo fu molto attiva presso l'Accademia Olimpica di Vicenza, malgrado non ne facesse ufficialmente parte; la sua frequentazione potrebbe stata esser connessa ai dibattiti riguardanti la scena teatrale che lì si tenevano. La donna ebbe senz'altro stretti rapporti con gli accademici; ciò è documentato non soltanto dai sonetti in suo onore composti da alcuni esponenti del prestigioso centro culturale (quali Angelo Ingegneri, Giovan Battista Maganza, Muzio Manfredi...), ma anche dalla lettera dedicatoria della Flori a Curzio Gonzaga in cui si accenna all'opera di supervisione dell'olimpico Paolo Chiappino. La scrittrice certamente fu in contatto con ulteriori figure di spicco del panorama letterario come Orsatto Giustinian, Luigi Groto (conosciuto col nome di Cieco d'Adria), Angelo Grillo, Bernardino Baldi e Torquato Tasso, il quale espresse stima per la Flori. ${ }^{8}$

\footnotetext{
${ }^{8}$ Scrive infatti il Tasso: "[i]o non poteva credere, che alcuno sentisse piacere d'esser vinto; ma leggendo la Favola Pastorale di V. s. con tanto diletto, ho conosciuto d'esser superato, e che niun vincitore si rallegrò più della propria vittoria; ma l'esser superato con tutti gli altri accresce il mio piacere, e la gloria di V. Sig. La ringrazio dunque, che m'abbia voluto far degno del suo dono, quasi di consolazione al cinto. E le bacio la mano. Di Roma il 12 d'Agosto del 1589." (TASSO 1738, lettera 506, p. 318).
} 
"Meglio ancora delle sue opere, che nessuno più legge": note per la riscoperta di Maddalena

Campiglia (1553-1595)

Anna Lisa Somma

Negli stessi anni, la vita della donna fu funestata da una dolorosa malattia agli occhi (menzionata in un paratesto della Calisa), nonché da liti a sfondo finanziario con i parenti.

Maddalena si spense il 28 gennaio 1595, a Vicenza. Prima di morire, affidò la cura dei suoi manoscritti a Orsatto Giustinian e a Curzio Gonzaga (ma, a quanto sappiamo, essi non furono pubblicati) e chiese infine nel testamento di essere tumulata nella chiesa di S. Maria Araceli delle clarisse francescane, "nello istesso loco et sepoltura dove si ha da sepellire il corpo della rev.da madre sor Giulia Cisotta, al presente benemerita abbadessa del convento d'Arcella" (MANTESE 1967, p. 113).

\section{Il Discorso sopra l'Annonciatione}

Se il Discorso sopra l'Annonciatione della Beata Vergine, e la Incarnatione del $S$ [ignor] N[ostro] Giesu Christo $(1585)^{9}$ a prima vista parrebbe soltanto una delle numerose opere devozionali del periodo, a una lettura attenta rivela stimolanti scarti a livello tematico, in grado di illuminare talune sfaccettature della condizione femminile negli ultimi decenni del XVI secolo, tanto più che è caratterizzato - secondo Lori J. Ultsch - dalla "denunciation of contemporary marriage practices and valorization of virginity" (ULTSCH (II) 2005, p. 353).

Primo lavoro di rilievo dopo la separazione dal marito e presumibilmente teso a favorire l'ingresso di Maddalena nella casta dei

\footnotetext{
9 Dal momento che non è ancora disponibile un'edizione critica del Discorso, tutte le citazioni qui riportate sono tratte dall'esemplare da noi consultato, custodito presso la Biblioteca Universitaria Alessandrina di Roma (Fondo Urbinate, segn. XIV e.16 8). Per la trascrizione del testo ci siamo attenuti a criteri conservativi (mantenendo dunque gli scempiamenti, le geminazioni, le grafie latineggianti, l'interpunzione dell'originale, etc.). Gli interventi normalizzanti hanno riguardato soprattutto la distinzione tra $\langle\mathrm{u}>\mathrm{e}<\mathrm{v}>$ secondo l'uso moderno e le abbreviazioni che interessano la resa delle nasali, della plosiva [p] e dell'occlusiva velare sorda seguita da dittongo ( $<$ qua $>,<$ que $>$, etc.), sciolte fra parentesi quadre.
} 
"Meglio ancora delle sue opere, che nessuno più legge": note per la riscoperta di Maddalena

Campiglia (1553-1595)

Anna Lisa Somma

letterati, per molti aspetti esso cercò di veicolare un'immagine conforme alla norma e stereotipata di lei, sia in quanto singolarità (donna rispettabile e pia, di saldi principi morali), sia in veste di auctor di sesso femminile che richiedeva per la sua arte il beneplacito di una platea maschile costituita da lettori e colleghi (o che, per lo meno nelle intenzioni di lei, erano da reputarsi tali).

I generi religiosi, d'altro canto, erano giudicati tra $\mathrm{i}$ più decorosi da coltivare per un'amante delle lettere, in quanto portatori di quei valori di pietas, castità, obbedienza e modestia sempre raccomandati con calore alle donne; di ciò la scrittrice era ben consapevole se nell'epistola dedicatoria a Curzio Gonzaga, nella Flori, scrisse:

So che le opposizioni [all'opera] saranno molte; ma di questa sola far dovrei stima, che fatto avessi meglio spendere il tempo in scritti spirituali, sì come avea cominciato, sviando la mente da qualunque vano pensiero [...]. (CAMPIGLIA 2004, p. 46)

Con accortezza, Maddalena optò nel Discorso per un tema già ben collaudato quale l'annunciazione, all'interno del quale, però, fu in grado di ricavare degli straordinari spazi di autonomia. Inoltre, per acquistare maggiore credibilità, tentò di dispiegare all'interno del testo tutte quelle conoscenze in materia religiosa che potevano giovare alla sua fama di erudita e devota: benché nella topica dichiarazione di modestia si proclami incapace e inadatta a trattare una materia così complessa e delicata, oltre agli immancabili accenni alle Sacre scritture, troviamo allusioni a Paolo, Dionigi Areopagita, Agostino, Gregorio e i "sacri Dottori", riservando persino un accenno alle pratiche di esegesi biblica. 
"Meglio ancora delle sue opere, che nessuno più legge": note per la riscoperta di Maddalena

Campiglia (1553-1595)

Anna Lisa Somma

Accompagnata da ben diciassette composizioni encomiastiche (redatte, fra l'altro, da Angelo Ingegneri, Giovan Battista Maganza e Luigi Groto) e da quattro sonetti di mano stessa dell'autrice - in cui, facendo professione di umiltà, innalza un conclusivo canto di lode a Maria -, l'opera è preceduta da un'epistola di Vespasiano Zugliano, In lode della Signora Maddalena Campiglia, che plaude l'animo retto della donna, e da una lettera dedicatoria indirizzata dall'autrice a suor Vittoria Trissina Frattina (1553-1612), nipote del più celebre Gian Giorgio. Quest'ultima, rimasta vedova giovanissima dopo soli tre mesi di matrimonio, divenne nel 1578 terziaria di san Domenico (pizzocara), per spegnersi poi circonfusa dalla fama di "santa viva".

Protagonista indiscussa del Discorso è, naturalmente, la Vergine, appena tredicenne, presentata nei giorni precedenti al suo ingresso nella casa di Giuseppe a Betlemme. Rimasta orfana di entrambi i genitori nello stesso giorno, ${ }^{10}$ distribuisce con larghezza le abbondanti sostanze ereditate da Anna e Gioacchino. Il suo sposo, Giuseppe, è rappresentato nelle vesti di giovane piacente - in netto contrasto con quanto riportato dalle credenze popolari, che hanno sempre attribuita al padre putativo di Gesù un'età avanzata - e di costumi irreprensibili ("non già mai nacque nel puro animo di costui un minimo pensiero d'incommodar i castissimi propositi di questa Santa Vergine, o violare i termini della Verginità sua": CAMPIGLIA 1585, p. 19).

L'attenzione dell'autrice è tutta concentrata sulla fanciulla, sull'incontro con l'angelo durante l'annunciazione e - com'è prevedibile - sulle lodi mariane. Proprio queste ultime procurano all'autrice il pretesto per instaurare un confronto con le sue contemporanee, fornendo un ritratto mosso (e a tratti

\footnotetext{
${ }^{10}$ Ciò non trova attestazione nei Vangeli $\mathrm{e}-\mathrm{a}$ quanto ci è dato sapere - neppure in quelli apocrifi.
} 
"Meglio ancora delle sue opere, che nessuno più legge": note per la riscoperta di Maddalena

Campiglia (1553-1595)

Anna Lisa Somma

gustoso, seppur approntato a dogmi rigoristici) delle loro abitudini, specie in materia religiosa: quanto la Madonna è umile, modesta, saggia e parca, finanche nei gesti più semplici, tanto, al contrario, le vicentine rinascimentali appaiono vanesie e volgari. ${ }^{11}$ Il culmine della contrapposizione fra onesta condotta morale e costumi quotidiani emerge con clamore in coincidenza delle sante funzioni, che si trasformano sovente in occasioni per esibire se stesse e i propri magnifici ornamenti, con l'intento di svettare nella piccola società di provincia. ${ }^{12}$

D'altronde, a giudizio di Maddalena, "Perseverantia, Mortificatione, Compassione, Prontezza, Ubidienza, Virginità, Fede, Humiltà, Fortezza, Chiarezza, Prudenza, \& Innocenza" (CAMPIGLIA 1585, pp. 18-19) sembrano essere le qualità richieste (e auspicabili) per una donna, propugnate dal modello mariano; eppure, le "giovani, maridate, \& vedove" - specchio antropico quasi perfetto della società muliebre del tempo - non vogliono e,

\footnotetext{
11 "Qui mi sovviene mentre Gabriello sta attendendo la risposta, \& Maria saggiamente discorrendo, ciò che risponder deve la creanza, il modo, l'humiltà, \& riverenza, con la qual'entriamo hoggi di noi altre donne, in Chiesa. Ohime ch'io veggio nell'entrar in Chiesa, nel salutar, nel render gli saluti, nel pigliar l'acque benedette, nell'humiliarsi al Sacramento Sacro \& Santo, nel star alle celebrate messe, nell'udir le predicationi, cosi poca divotione, tanta alterezza, \& orgoglio, cosi sconcia e sgarbata maniera di creanza [...]." (CAMPIGLIA 1585, p. 40).

12 "Ma hoime che hò veduto io (\& per avventura il peggio sono tra queste) molte, le quali à pena la Dominica entrate in chiesa per udir Messa, overo à Vespero il giorno à caso, à quella con poca divotione stanno ragionando sempre o degli affari suoi, ò di qual che mica di pochissima importanza, \& apena un ginocchio solo piegano per riverenza: \& al Vespero poi poco, e nulla attendono à ciò che si dice nelle lodi cantate à Dio ne' Chori de sacerdoti: solamente attendendo à consigliarsi in trovar luochi per andar à diporto; ov'a pena fornito il Vespero fattessi venir le loro carroccie, un signacolo per usanza si fanno, \& a caso piegate un poco anzi niente se n'usciscono di Chiesa cosi atediate di quella, come se mill'anni fossero, che ivi soggiornato havessero chi qua, chi là spargendosi vestite di mille varij colori, adorne di perle, \& cathene con mille fregi e novelluccie dintorno, \& servitori, e paggi a piedi andando per la Città, e vansi porgendo per idolo a cui le fanno incontro. [...] Giunte a casa poi queste signore giovani, maridate, \& vedove (che d'ogni sorte di queste parlo io hora) pongono ogni lor cura in riserbar i levati adornamenti suoi per il giorno sequente senza sconciarli ò d'imbruttarli punto, \& con'ogni studio loro attendono per comparire novamente con nove foggie, \& garbature à concorrenza dell'altre, e far nuovi discorsi per trovar ricami, \& abbigliamenti moderni. Questo è l'amore, queste son dunque le brame ch'hoggidì si ritrovano nel cor di noi altre donne verso Dio nostro Signore?” (CAMPIGLIA 1585, p. 47).
} 
"Meglio ancora delle sue opere, che nessuno più legge": note per la riscoperta di Maddalena

Campiglia (1553-1595)

Anna Lisa Somma

per taluni versi, non riescono ad adeguarsi alle virtù su elencate, anche a causa di uno stile di vita che le rende "troppo attente, \& immerse [...] negli strepiti famigliari, \& basi” (CAMPIGLIA 1585, p. 56). Ciò - è possibile intuire potrebbe esser ricollegabile a uno status matrimoniale problematico e, purtroppo, alquanto comune; dopo tutto, nelle parole della Campiglia è arduo non tentare di individuare, celati dietro i motivi dello scontento generale, riflessi di esperienze personali.

A questo proposito è interessante esaminare le caratteristiche del legame nuziale di Maria e Giuseppe, paragonandolo a quello diffuso alla fine del Cinquecento, criticato, quest'ultimo, con una certa acredine dall'autrice. Tra le due sante figure pare intercorrere un rapporto paritario, più volte ribadito sin dall'incipit stesso, che quasi prefigura il sodalizio tra Flori e Alessi nella favola pastorale di là da venire. Giuseppe infatti dichiara:

\footnotetext{
Et, poi che ho io da Iddio cosi gran favore ricevuto d'esserti compagno, a me so quanto conviene di fare; però ad'ogni picciolo tuo cenno farà con'ogni poter mio essequito il commandamento, \& voler tuo. Havuta ch'hebbe Maria Santa questa amorevol, \& dolce risposta, conchiuse seco anci postponendo sempre il suo al voler di lui [...]. (CAMPIGLIA 1585, pp. 4-5)
}

E ancora, più specificatamente, viene detto:

\footnotetext{
In questo consenso de gli animi loro s'effettuò veracemente il matrimonio, poiche con questa mutua virginità in mutua servitù consolati, si posero ambi in obligo espresso di mutua, \& perpetua fede; non altro mirando, che mantener con il favor di Dio inviolata la purità verginale dell'anima, \& del corpo questa più prezzando, che non sogliono far'i maggior Regi la prole, \& posterità loro. (CAMPIGLIA 1585, p. 7)
} 
"Meglio ancora delle sue opere, che nessuno più legge": note per la riscoperta di Maddalena

Campiglia (1553-1595)

Anna Lisa Somma

Un connubio ideale, al punto che "senza pari in terra è stata la fortunata sposa di Giuseppe" (CAMPIGLIA 1585, p. 26); sicuramente molto diverso dagli sfortunati legami che Maddalena Campiglia, con buone probabilità memore della sua storia, descrive:

O' benedetto, \& sacro santo Matrimonio che cosa non puoi tu co'l favor d'Iddio? \& quando massimamente egli avviene, che concordi, \& pari siano gli animi de' giugali. All'hora veramente ciò avviene, quando l'una parte, \& l'altra voti gli animi di malignità coperta con'un cor non già ferigno, \& pieno di tosco, ma humile, \& tutto caldo dell'honor d'Iddio, del mondo, e di lor medesmi, attendono insieme à far vita Santa, e buona, facendo del voler dell'uno e l'altro legge a se stessi. Ma ò quanto sono diversi gli usi di tali à questi tempi nostri, ove non altro si scorge, o intende, che crudeli riffe, inauditi rancori, empie parole, buggiarde calonnie, \& scelerate attioni. (CAMPIGLIA 1585, p. 6)

Un'asprezza che sfiora il livore gonfia le parole di Maddalena ed esse emergono con inaspettata vis drammatica, lenita solo dal successivo ricorso all'usuale, lussureggiante repertorio di lodi mariane, che pur nasconde appena altri strali anti-matrimoniali:

O eccellentissima donna, Vergine sopra tutte le Vergini, saggia, \& felice, adorna di fregi cosi sublimi, \& risplendenti. Non cinta ti veggo di catena di ferro grave, \& pesante, come le maritate, che sotto l'insopportabil peso del matrimonio in modo sono gravate, ch'in dispetto di lor medesme tal'hor ne vengono, \& bene spesso odiano quest'aria, che le spira d'intorno: Ne meno di collane d'argento fino, \& terso ti miro adorna ò Sacra Regina mia, qual ne va altero il stato vedovile, ma di purissimo oro freggiata ti scorgo qual il merto della Verginità tua ricerca. (CAMPIGLIA 1585, p. 23) 
"Meglio ancora delle sue opere, che nessuno più legge": note per la riscoperta di Maddalena

Campiglia (1553-1595)

Anna Lisa Somma

L'avversione di Maddalena Campiglia all'istituto nuziale pare configurarsi sia come necessità di aree autonome, sia come decisa aspirazione verso quei valori di castità e rigore morale cui Maria aderì in maniera così perfetta in vita; castitas, in questo caso, liberamente scelta e perseguita, "non riducibile alle valenze dell'bonos, né alle prescrizioni dell'institutio foeminae christianae" (CHEMELLO 2003, p. 86).

Del resto, è proprio nel Discorso che, secondo Carlachiara Perrone, sarebbe possibile rinvenire i prodromi del rifiuto di servire all'uomo (poi formalizzato, come vedremo, anche a livello verbale esplicito, nella Flori), sotto forma di celebrazione della condizione virginale da intendersi come "somma perfezione spirituale" (CAMPIGLIA 1996, p.38).

\section{La favola pastorale Flori}

Insieme alla Mirtilla di Isabella Andreini (1588), la Flori costituisce uno dei rari esemplari di drammi pastorali composti da donne nella prima età moderna. Pubblicata nel 1585, essa segna una svolta nel percorso scrittorio di Maddalena, che abbandona il genere devozionale per consacrarsi a quello bucolico - allora molto in voga sulla scorta dell'Aminta tassiano -, dandone una rilettura assolutamente personale, in parte autorizzata dal fatto che, nella pratica, la favola boscareccia (come era allora definita) non possedeva una tradizione classica cui rifarsi (il genere, infatti, era nato nel corso dell'Umanesimo).

Secondo Virginia Cox, qui Maddalena Campiglia avrebbe tentato di esplorare la possibilità di una creatività muliebre indipendente dalla giurisdizione maschile; capovolgendo la consuetudine secondo la quale sotto 
"Meglio ancora delle sue opere, che nessuno più legge": note per la riscoperta di Maddalena

Campiglia (1553-1595)

Anna Lisa Somma

le rozze vesti dei pastori si nasconderebbero praticanti della poesia, nella Flori questo ruolo spetterebbe a pieno titolo alla sua protagonista, una ninfa. ${ }^{13}$

Sebbene il plot della Flori si adegui formalmente ad alcuni capisaldi del genere - adottando elementi quali la ritrosia delle pudiche eroine, gli amori pastorali non corrisposti, le immancabili agnizioni, le nozze e i colpi di scena risolutori... -, esso presenta delle peculiarità degne di nota. Mettendo da parte i fili narrativi secondari, dedicati per lo più alle vicende dei personaggi minori, al centro dell'opera troviamo l'omonima ninfa Flori, seguace di Diana e invaghita castamente della bella Amaranta, purtroppo spirata tempo prima; a nulla valgono le preghiere e gli ammonimenti dell'amica Licori a liberarsi da questa passione. Flori sembra impazzita d'amore, parimenti ad Androgeo, follemente infatuato di lei, che sprezza i suoi sentimenti. Ciononostante,

Il negarsi della vergine al rapporto con l'uomo, tradizionalmente unico desideratum femminile, non è negazione del desiderio, al contrario, la ninfa Flori ha un desiderio fortissimo, che si manifesta in due forme: la prima è quella della dedizione al proprio alto ideale fuori dal più comune destino femminile, tendendo quindi alla produzione di opere dell'ingegno, piuttosto che di figli: "ad alto spera ch'altera sprezza questa Ninfa il calle da 'l comun piè donnesco impresso, e poggia per solitaria strada a mercar lode" (Flori, V). La seconda è il desiderio amoroso rivolto ad un'altra donna che, a differenza di quello per l'uomo, non sembra ostacolare la tensione ideale e l'ambizione femminile, consentendo la salvaguardia dell'autonomia e della verginità. (SARTORI 1994, p. 62)

L'insanità di Flori è dunque di natura ben diversa rispetto a quella di Silvia nell'Aminta: se quest'ultima - acerba emotivamente e, forse, sessualmente - è definita "pazzarella" (TASSO 1952, v. 99) poiché rifiuta di sottostare all'amore (inteso come forza naturale che governa l'universo) e

${ }^{13}$ Per approfondimenti cfr. Cox (2008, p. 158). 
"Meglio ancora delle sue opere, che nessuno più legge": note per la riscoperta di Maddalena

Campiglia (1553-1595)

Anna Lisa Somma

fugge dalle responsabilità procreative per dedicarsi alla caccia, l'eroina della Nostra non rigetta in toto il sentimento amoroso, bensì anela all'“impossibil" (atto V, scena I, v. 54) e al "vano", ${ }^{14}$ in quanto innamorata di una donna defunta, e per di più aspira a una fecondità non biologica, ma intellettuale (come vedremo a breve). Le sue emozioni non sono condivise dai personaggi di scena, che le reputano sterili, incomprensibili, inutili.

Giunti a questo punto, ci sembra opportuno sottolineare quello che, apparentemente, parrebbe solo un riuscito escamotage per la costruzione del lieto fine, invero gravido di implicite conseguenze. Per riacquistare il senno perduto, Flori e Androgeo prendono parte a un rituale per liberarsi dall'amore folle, sottoponendosi a quello che si potrebbe assimilare a un rito di passaggio dalla giovinezza all'età adulta (e dunque dalla libertà - persino sconsiderata e/o trasgressiva - delle emozioni alla loro irreggimentazione e alla normalizzazione dei ruoli sociali, nonché di gender). Una volta sciolto dall'incantamento, il pastore è libero di eleggere la compagna di vita che preferisce. Flori, al contrario, a causa di un nuovo sortilegio, è costretta a concepire amore per il primo uomo che incontrerà: come non di rado accadeva nella società contemporanea all'autrice, la donna non ha possibilità di decidere in accordo ai suoi desideri, bensì deve sottostare ai voleri di un'entità superiore, la quale agisce senza curarsi della felicità o delle ambizioni della diretta interessata.

Durante la cerimonia, cui partecipa tutta la comunità, Flori ha occasione di conoscere e innamorarsi all'istante del "pastore straniero" Alessi, il quale non può che ricambiare. Non li attende, tuttavia, al pari delle altre coppie che affollano la scena, un'unione convenzionale e pacificante. Per loro

\footnotetext{
${ }^{14}$ L'attributo "vano" ritorna sovente nel testo, non solo in connessione alla passione di Flori per Amaranta: sono infatti definiti "vani" gli amori smodati e non corrisposti - omo ed eterosessuali che siano -, che producono vaneggiamento e follia.
} 
"Meglio ancora delle sue opere, che nessuno più legge": note per la riscoperta di Maddalena

Campiglia (1553-1595)

Anna Lisa Somma

stesso volere, il nodo matrimoniale che li stringerà insieme sarà fondato su una mutua eguaglianza e una castitas che permetterà alla donna di dedicarsi alla cura e allo sviluppo del suo ingegno, anziché della prole, in accordo a quanto già da lei dichiarato a Licori: "Sian nostri figli le cose create/ dal divino nostro pelegrino ingegno,/ né serva ad uomo angelica fattura" (atto V, scena III, vv. 90-92).

Il rapporto del tutto peculiare fra Alessi e Flori ne fa quindi una "coppia [...] unqua veduta" (prologo, v. 61), eccezionale in ogni senso, che accondiscende a plasmarsi sulle aspettative del polo femminile. In quanto accolita di Diana, infatti, la ninfa non sarebbe autorizzata a legarsi a uomo (né, d'altro canto, vorrebbe trasgredire il divieto); accetta di contrarre gli sponsali solo a condizione che non infrangano i suoi alti precetti morali:

Lieta allor potrei star ch'Alessi meco dedicandosi a Cinzia castamente, di mutuo nodo avinto, in pari fiamme ardesse meco; allora ben sarei lieta. (CAMPIGLIA 2004, atto III, scena IV, vv. 228-232)

Del resto è ribadito a più riprese che Flori e Alessi si amano in “disusato modo". È utile soffermarsi sulle occorrenze testuali della locuzione (e, in un sol caso, di "disusati ardori”): essa è sempre accompagnata nei versi adiacenti da riflessioni sulla natura dell'amore della ninfa per Alessi, e sempre in connessione all'ardore e alle fiamme di una passione castissima. ${ }^{15}$

\footnotetext{
15 "Non più celare, o Flori, al tuo pastore/ de l'onesto tuo cor la pura fiamma./ Volgi le luci in quelle luci amate,/ che dianzi mi dicevi/ che in disusato modo ardèanti l'alma [...]" (Licori, atto V, scena II, vv. 73-77); 'T'amo, Alessi, no 'l nego,/ in disusato modo./ De' tuoi begli occhi il pianto/ ch'estinguir dovea 'l foco/ esca fu a le mie fiamme./ Ma tali son queste mie fiamme pure/ ch'ardendo non consumano, sì tempra/ l'ardor di pudicizia umor soave." (Flori, atto V, scena II, vv. 85-92); “Ahi, ch'ora avien ch'altri mi leva in tutto/ di poter di me stessa più disporre./ Questo pastore [: Alessi], Androgeo, questo, questo/ m'ha furato dal petto l'alma e 'l core,/ e in disusato modo or tutta m'arde." (Flori, atto V, scena III, vv. 158-162); "Poc'or non disse Flori/ che le lagrime
} 
"Meglio ancora delle sue opere, che nessuno più legge": note per la riscoperta di Maddalena

Campiglia (1553-1595)

Anna Lisa Somma

I sentimenti di Flori sono immacolati e sbocciati dalla pietà, capaci di temprare l'animo al pari di un fuoco purificatore:

[Flori] rivolgendosi ad Alessi gli confessa un amore che si manifesta in un "disusato modo", perché la sua fiamma ha un effetto catartico verso il passato (dovendo estinguere il fuoco dell'amore verso l'estinta Amaranta) e rigeneratore verso il futuro. La nuova fiamma d'amore (verso Alessi) non solo è destinata a non consumarsi ma, come l'araba fenice, sembra rigenerarsi dalle proprie ceneri, ad indicare allusivamente la fiducia in una rinascita destinata a realizzarsi fuori del tempo e dello spazio. Una rinascita che resta ancorata ad un presente, a quel nodo amoroso che trattiene in sé le marche della onestà, santità e castità [...]. Presentandosi come una "nova coppia d'amanti", Flori e Alessi propongono contestualmente un "novo modo d'amar" che, accanto le ritrosie e le resistenze della ninfa, supera l'opposizione tra il culto di Diana e quello di Amore per una realizzazione sincronica che li comprenda entrambi. (CHEMELLO 2003, p. 95)

Il "disusato modo" nuziale propone insomma un paradigma matrimoniale alternativo che prevede un rapporto paritario tra $\mathrm{i}$ coniugi, non vòlto alla procreazione e per giunta fondato sul rispetto di valori quali la castità e la stima reciproca (e dunque sensibilmente diverso da quello sancito dalla consuetudine nella prima età moderna): un modello che non può non rammentare il legame ideale già prefigurato nel Discorso, quello tra Maria e Giuseppe.

Il tema della fecondità intellettuale, anziché biologica, ritorna con decisione anche nelle due lettere dedicatorie della Flori, l'una indirizzata a

tue/ entro al sen le destaro/ con disusato modo il foco ond'arde?" (Androgeo rivolto ad Alessi, atto V, scena III, vv. 212-215); "I disusati ardori/ che t'apportano al cor gradito affanno/ tempra, ch'alta mercede/ dal tuo ALESSI s'aspetta a la tua fede." (Maddalena Campiglia alla sua Flori). Tutti i corsivi sono nostri. 
"Meglio ancora delle sue opere, che nessuno più legge": note per la riscoperta di Maddalena

Campiglia (1553-1595)

Anna Lisa Somma

Isabella Pallavicino Lupi (nobildonna e, forse, patrona dell'autrice), l'altra a Curzio Gonzaga, poeta e amico, legandosi strettamente alla rivendicazione di un riconoscimento sociale e culturale.

La strategia di Maddalena in questi scritti paratestuali è piuttosto chiara: nel momento in cui esibisce le proprie mancanze (o quel che è passibile di esser ritenuto tale) - anticipando così i rimproveri e dando prova di una profonda dimestichezza con i meccanismi del sistema letterario, tanto nell'ambito dei genre e della teoria, quanto in quello della pratiche sociali -, dimostra anche le sue conoscenze e la sua perizia (a livello di tecnica, teoria letteraria, lingua, familiarità con gli auctores...), pur dissimulandole dietro topiche dichiarazioni di modestia e invocazioni di protezione. Grazie a ciò, può reclamare con orgoglio il suo essere donna e intellettuale e mettere quindi da parte ruoli tradizionali - quali quello di generatrice biologica e scrittrice religiosa -, per farsi madre letteraria e autrice che non teme di affrontare generi prettamente di dominio maschile, affermando in tal modo se stessa al di là delle convenzioni.

Come accennato innanzi, la donna estrinseca in modo puntuale e con una sottilissima, carsica ironia tutti i presunti punti deboli della Flori (per lo meno, in un'ottica maschile). Innanzitutto, spiega a Gonzaga, occorre cautelarsi dagli uomini maldisposti a priori nei confronti delle scritture femminili, per vizio dell'animo o dell'intelletto:

Ma sopra tutto la gentilezza del cortesissimo animo di Vostra Signoria Illustrissimo m'ha dato sicurezza, nonché speranza, ch'ella sia per difender questo mio poema pastorale da tutti quelli del sesso virile, $i$ quali se ne scopriranno detrattori, o per maligna disposizione o per 
"Meglio ancora delle sue opere, che nessuno più legge": note per la riscoperta di Maddalena

Campiglia (1553-1595)

Anna Lisa Somma

abuso di sinistro giudizio contra i componimenti poetici delle donne.

(CAMPIGLIA 2004, p. 46)

Significativamente, infatti, al fondo delle due epistole troviamo - rileva Philiep Bossier - una triplice poetica alternativa: una poetica dell'anticipazione, che mira a tutelare Maddalena dalle critiche; una poetica dell'autonomia, che "prova a rompere l'automatismo dei luoghi comuni appartenenti a un genere" (BOSSIER 2007, p. 55) e, infine, una poetica della libertà, legata alla rivendicazione della propria autonomia nel comporre. La Nostra è insomma ben consapevole di trasgredire sua sponte le norme comunemente accettate, su tre distinti piani (in ordine di occorrenza testuale: autoriale e morale, teoricoletterario, stilistico). Per ciascuno di essi evoca uno o una serie di personaggi noti facenti funzione di numi protettori - sant'Agostino, i grandi autori che pur non hanno seguito pedissequamente la Poetica aristotelica, il letterato contemporaneo Paolo Chiappini -, capaci di porre sotto la loro egida benevola l'operato della poetessa attraverso la loro parola o la loro azione, in maniera diretta o indiretta. Nel fingere contrizione per gli errori, Maddalena in realtà rivendica la propria originalità e il proprio valore:

\footnotetext{
So che le opposizioni saranno molte; ma di questa sola far dovrei stima, che fatto avessi meglio spendere il tempo in scritti spirituali, sì come avea cominciato, sviando la mente da qualunque vano pensiero; se da Sant'Agostino data non me ne fosse licenza con affermar che ogni sorte di virtù allontana l'uomo dai vizî. (CAMPIGLIA 2004, p. 46)
}

Dimostrate appieno le sue conoscenze (in grado di tener testa a quelle dei colleghi di sesso maschile), la scrittrice può rivendicare con fierezza la paternità del testo, anzi: la maternità. 
"Meglio ancora delle sue opere, che nessuno più legge": note per la riscoperta di Maddalena

Campiglia (1553-1595)

Anna Lisa Somma

Madre sollecita, nel dedicare la favola pastorale a Isabella Lupi Pallavicini, Maddalena si premura di affidarle l'opera-figlia affinché venga tutelata dalle maldicenze. Nel far ciò confida nell'animo "virile" della nobildonna, capace di apprezzare gli originali frutti di un ingegno muliebre fuori dalla norma:

Sono tuttavia sicurissima che, sendo ella tanto virile nei pensieri e nelle operazioni quanto donna nel bellissimo sembiante e negli onestissimi portamenti, aggradirà questo mio rozzo parto, e la viva candidezza del cuore con che lo accompagno. Sogliono tutte le madri d'oggidì, dovendo far comparir fuori le loro figlie, comporle nella più leggiadra maniera che si sanno imaginare, ricercando a questo effetto i più riposti e astrusi cantoni dell'arte; il che a me non giova di fare, procurando più tosto d'allontanarmi dall'ordinario costume donnesco. Miri ella dunque non con l'occhio della serena sua fronte in questa mia figlia estrinseca pompa di vanità volgare (che essendo nata fra' boschi ha dalla madre imparato a sdegnar i politici addobbamenti) [...]. (CAMPIGLIA 2004, p. 44)

Il ricorso alla metafora del parto non è adoperato solo in virtù di una presunta solidarietà muliebre che si instaurerebbe tra la poetessa e la marchesa; la figura retorica, infatti, ritorna nella dedica seguente, destinata a Curzio Gonzaga (e, anche in questo caso, con lo scopo di richiedere protezione):

Poiché non le posso dar cosa più cara di questa mia figlia, vera figlia, e naturale, di che prencipalmente mi godo. Perciò che se io la vedrò benignamente accolta da lei, m'andrò preparando per porgerle maggior segno della divozione ch’io serbo al suo chiarissimo valore. (CAMPIGLIA 2004, p. 48)

I consistenti riferimenti alla maternità letteraria nei due testi preliminari collocano inequivocabilmente l'opera sotto il segno di un femminino che rifiuta la maternità biologica e la sottomissione all'uomo ("È vero/ Ch’io 
"Meglio ancora delle sue opere, che nessuno più legge": note per la riscoperta di Maddalena

Campiglia (1553-1595)

Anna Lisa Somma

lodai, lodo, e loderò mai sempre/ Il non servire ad uomo, che d'uomo ha solo/ La sembianza, onde copre insane voglie/ Spesso, e di mostro e fera ingegno e mente.", atto V, scena III, vv. 100-104) per consacrarsi invece alla generazione poietica (intesa nel doppio senso di creatrice di vita e di poesia), libera e indipendente. ${ }^{16}$

D'altro canto, parrebbe confermare ulteriormente questa vena demiurgica lo stesso appellativo di Flori, il quale rievoca quello della dea romana (già italica) Flora, custode della potenza vegetativa che presiede le fioriture. Rapita dal dio del vento Zefiro, di lei innamorato, divenne sua sposa e da lui fu investita "mater florum", come illustrato nella Primavera di Botticelli. Il mito è narrato anche da Ovidio nei Fasti (in cui la ninfa figura col nome Clori $)^{17}$ e lo scrittore propone qui un racconto che ci sembra in linea con la volontà, da parte di Maddalena Campiglia, di presentare perfino onomasticamente la sua protagonista quale lodevole esempio di indipendenza femminile. Clori, difatti, avrebbe aiutato Giunone - irata per la nascita spontanea di Minerva dal capo di Giove - a concepire senza un tramite maschile un figlio, Marte, facendole assumere così lo status di "sine coniuge mater". ${ }^{18}$ Esattamente come l'autrice in rapporto alla sua opera.

\section{L'egloga Calisa}

\footnotetext{
${ }^{16}$ Una simile volontà di autodeterminazione è rintracciabile anche in un sonetto (pronunziato per bocca di Corinna) nel contemporaneo Il merito delle donne di Moderata Fonte: "Libero cor nel mio petto soggiorna,/ non servo alcun, né d'altri son che mia,/ pascomi di modestia, e cortesia,/ virtù m’essalta, e castità m’adorna.// Quest'alma a Dio sol cede, e a lui ritorna,/ benché nel velo uman s'avolga, e stia;/ e sprezza il mondo, e sua perfidia ria,/ che le semplici menti inganna, e scorna.// Bellezza, gioventù, piaceri, e pompe,/ nulla stimo, se non ch'a i pensier puri,/ son trofeo, per mia voglia, e non per sorte.// Così negli anni verdi, e nei maturi,/ poiché fallacia d'uom non m'interrompe,/ fama e gloria n'attendo in vita, e in morte.": Moderata Fonte (1988, pp. 18-19) riportata in Chemello (2003, pp. 100-101).

17 "Chloris eram, quae Flora vocor: corrupta Latino/ Nominis est nostris littera Graeca sono." (OVIDIO 1998, vv.195-196).

${ }_{18}$ Per l'episodio si veda Ovidio (1998, vv. 229-256). Per ulteriori approfondimenti sulla figura della ninfa, cfr. la voce Flori in Grimal (1994, pp. 291-292).
} 
"Meglio ancora delle sue opere, che nessuno più legge": note per la riscoperta di Maddalena

Campiglia (1553-1595)

Anna Lisa Somma

L'anno successivo alla comparsa della Flori Campiglia diede alle stampe Calisa, egloga pastorale composta per celebrare lo sposalizio di Giovan Paolo Lupi (figlio di Isabella Pallavicino Lupi, qui presentato con l'appellativo pastorale etimologico Lico) e Beatrice degli Obizzi (Bice). Similmente all'opera precedente, il mondo bucolico fornisce all'autrice l'occasione per portare allo scoperto elementi trasgressivi e/o polemici, uniti a forte istanze di autonomia creativa.

Come apprendiamo dalla lettera dedicatoria (datata 9 novembre 1589), anche questo scritto è posto sotto la tutela di Curzio Gonzaga. Esso sarebbe stato edito ancora imperfetto, contro la volontà dell'autrice, approfittando di una sua indisposizione agli occhi e di un furto. Ricorrendo all'excusatio, Maddalena non soltanto afferma latamente di aver pubblicato l'editio princeps dell'opera sotto il suo diretto controllo, ma "ammettendo implicitamente la probabilità di «errori» imputabili a [sé] stessa [...] [conduce] quella rivendicazione della libertà di scrivere senza l'assillo di una perfetta imitazione che «era, fuori di Toscana, ovvia e frequente»" (STUSSI 1993, p. 151 cit. in CAMPIGLIA 1996, p. 91), mettendosi preventivamente in guardia dalle critiche del pubblico (specie se maschile) dietro la topica professione di modestia.

Anticipando le possibili rimostranze a livello teorico-letterario (“[...] ragion vuole che di gran lunga un'egloga sia inferiore ad una favola": CAMPIGLIA 1996, p. 71), la scrittrice dimostra nuovamente di esser ben padrona degli strumenti critici e retorici, evidenziando al tempo stesso a Gonzaga: 
"Meglio ancora delle sue opere, che nessuno più legge": note per la riscoperta di Maddalena

Campiglia (1553-1595)

Anna Lisa Somma

$[\mathrm{N}]$ on sono (mercé sua) conosciuta immeritevole di perpetuamente servire all'infinito suo valore, potrò ben stimare da qui inanzi ogn'azzion mia piu che finora non abbia fatto e quindi tener piu degna Calisa che Flori. ${ }^{19}$ (CAMPIGLIA 1996, p. 71)

Protagonisti indiscussi della breve operetta (291 versi in totale) sono Edreo (plausibilmente alter ego del letterato cesenate Muzio Manfredi), severo pastore, e la già nota Flori. Malgrado siano riuniti per celebrare l'unione di Lico e Bice, i due finiscono per porre al centro delle loro discussioni la liceità e la natura dell'amore saffico che la ninfa nutre per Calisa, ${ }^{20}$ sorto in Flori dopo l'improvvisa scomparsa del promesso sposo Alessi ("Deh, caro Alessi mio, ove or ti stai?", v. 163): segno, questo, che il matrimonio all'insegna del "disusato modo" adombrato nella favola pastorale si è forse rivelato fallimentare o d'impossibile realizzazione.

L'opera principia con un discorso colto in medias res: Edreo, sicuro di sé, assicura che riuscirà a far mutare opinione a Flori ricorrendo alla sua eccellente oratoria ("Et io lo saprò far, ch'ingegno et arte/ e parole ho ben atte!", vv. 1-2). Egli, infatti, facendosi beffa degli amanti omosessuali, vorrebbe:

con mie ragioni et arti lei ritrarre da questi vani et infelici amori. (CAMPIGLIA 1996, vv. 28-36)

L'uomo, dunque, ribadisce come intenda porre al servizio del suo obiettivo tutti gli strumenti di cui dispone, in termine di intelletto ("ragioni") e

${ }^{19}$ Ciò, d'altro canto, costituisce una sorta di tacita controprova della bontà del suo lavoro.

${ }^{20}$ Nella prima parte dell'opera trovano posto anche le lodi di alcuni membri del cenacolo stretto intorno a Calisa, quali il Regio pastor gentile (Curzio Gonzaga), la Felice Orsa (la duchessa di Paliano Felice Orsina Colonna, dedicataria del Fidamante) e il Pastor Divo (il cardinale Scipione Gonzaga); la seconda sezione, invece, è maggiormente improntata al canto nuziale. 
"Meglio ancora delle sue opere, che nessuno più legge": note per la riscoperta di Maddalena

Campiglia (1553-1595)

Anna Lisa Somma

mezzi tecnici ("arti”); le sue parole e la sua determinazione - ne è convinto riusciranno a opporsi alle "speranze e [a]i detti/ vani”" (vv. 61-62) della donna.

Nonostante la ninfa arda castamente per Calisa, madre di Lico, il pastore - in preda al disappunto - cerca a più riprese di persuaderla ad abbandonare la sua insana passione: non soltanto la sua "calda brama" (v. 81) è disdicevole e folle, dal momento che lei erra "soverchio/ donna amando pur donna essendo" (vv. 99-100), ma concepisce un amore inutile, destinato a condurla a morte sicura.

I tentativi di Edreo, però, non giungono a buon fine dinanzi alla tenacia della donna; al contrario, nel difendere la purezza dei sentimenti che ha concepito, Flori riconosce la sua umana fragilità e reclama con forza la possibilità di amare alla propria maniera, per quanto inconsueta:

So che donna amo donna, ahi, ch'anzi adoro ninfa umile una dea celeste in terra, ma INFERMO gusto al suo peggior s'appiglia, né dritto sceme occhio ch'è infermo; e poi CHI pon freno agli amanti, o dà lor legge?

Già cieca son d'amor, già vinta giace la ragion, né 'l discorso ha in me piu loco.

Nel tempio del mio cor l'alma CALISA mio NUME è fatta et idol mio sovrano, a cui già offerto ho le potenze e i sensi e da lui preso ogni decretto e legge. Com'ella vuole e vuole Amor mi reggo, né a me piu giova libertade o pace di quel che dare a me sia loro in grado. (CAMPIGLIA 1996, vv. 104-117) 
"Meglio ancora delle sue opere, che nessuno più legge": note per la riscoperta di Maddalena

Campiglia (1553-1595)

Anna Lisa Somma

Edreo, battuto dalle ragioni e dalla facondia della ninfa, è costretto a desistere dai suoi intenti e a confessare che Calisa contraccambia i sentimenti. ${ }^{21}$ Malgrado tenti di nascondere la propria sconfitta dando la colpa all'insanabile condizione della fanciulla, afflitta da "fatal piaga" (v. 119), l'uomo è ben conscio delle potenzialità intrinseche di Flori:

Dunque, ama, Flori, e spera: un giorno forse, benché strano è 'l tuo amor, ne corrai frutto.

(CAMPIGLIA 1996, vv. 124-125)

Mentre, a dispetto delle aspettative, il canto del pastore non ha sortito l'effetto sperato, è proprio l'amore "strano" di Flori per Calisa a dar frutti: il primo e più saporoso è certo costituito dall'egloga medesima. Se il poeta Edreo, convinto di poter mutare il pensiero di Flori, subisce una pesante sconfitta, la ninfa conquista invece una doppia vittoria, poiché vede riconosciuti sia il diritto a un amore altro, sia l'efficacia della sua parola. Grazie a un sapiente uso dell'arte retorica, la ninfa riesce difatti a focalizzare l'attenzione sul suo desiderio non convenzionale (giungendo addirittura a convincere Edreo, almeno in parte, della liceità di un amore omosessuale), scalzando così il tema principe dell'egloga, vale a dire il panegirico del matrimonio eterosessuale.

Giunta al termine di un cammino inaugurato nella favola boschereccia e che l'ha vista alle prese con numerose peripezie (il grave lutto causato dalla perdita di Amaranta, una passione "vana" per lei, un amore "disusato" seguito

\footnotetext{
21 "Già raccolto e gradito ha 'l cor, l'affetto/ la tua bella CALISA, e sua ti chiama/ in ripa d'Adria ragionando meco./ «La mia Flori», sovente ella dicea/ e le piacea mirar tue note incise." (CAMPIGLIA 1996, vv. 130-134).
} 
"Meglio ancora delle sue opere, che nessuno più legge": note per la riscoperta di Maddalena

Campiglia (1553-1595)

Anna Lisa Somma

da un matrimonio egualitario ma fallimentare...), Flori nella Calisa sembra finalmente esser riuscita a raggiungere lo scopo che si era proposta già a partire dall'opera precedente, vale a dire conquistare la gloria poetica, seguendo così le orme di Vittoria Colonna:

[...] tenta meco poggiar per quel sentiero ove donna immortal sola sen gìo

VITTORIOSA e DIVA tra mai quante del sesso nostro fur chiare e illustri. (CAMPIGLIA 2004, atto I, scena I, vv. 105-108)

\section{Considerazioni finali}

Come speriamo si sia potuto appurare dal nostro lavoro, il profilo e l'opera di Maddalena Campiglia appaiono del tutto peculiari nel panorama tardo rinascimentale italiano per scelte tematiche, consapevolezza teorica e, non in ultimo, spunti polemici.

Essi - celandosi di frequente sotto il velo della rassicurante finzione bucolica - mirarono a porre in discussione alcuni elementi ideologici e sociali fondanti dell'epoca, quali l'istituzione matrimoniale, il consueto e angusto ruolo della donna (procreatrice, madre e moglie), nonché gli spazi a lei riservati ( $\mathrm{o}$, al contrario, preclusi) per la sua definizione e la sua affermazione intellettuale. La stessa, presumibilmente difficoltosa, esistenza della scrittrice condotta all'insegna di una costante rivendicazione di autonomia - non poté che confermare un impegno radicato e costante nel tempo nello sfidare cliché e rigide tassonomie sul duplice piano del gender sessuale e del genere letterario.

Per questo insieme di motivazioni ci pare opportuno respingere almeno in parte il paradigma esegetico forse tuttora dominante (o che, perlomeno, è 
"Meglio ancora delle sue opere, che nessuno più legge": note per la riscoperta di Maddalena

Campiglia (1553-1595)

Anna Lisa Somma

stato a lungo tale) applicato alle creazioni della Campiglia, ispirato a una prospettiva limitatamente spirituale-religiosa, per dialogare invece con modelli più flessibili, capaci di abbracciare una complessità cangiante e poliedrica, che trova una sua significativa continuità nel corpus testuale.

Ciò vale in primo luogo per il trattamento delle tematiche omosessuali, plausibile principale fonte di fraintendimenti riguardanti la figura e gli obiettivi dell'autrice: a differenza di quanto avvenuto in larga parte della produzione coeva (specie comico-umoristica), ${ }^{22}$ nella Flori e nella Calisa questi motivi non sottostanno a finalità ludiche (alla stregua di un divertissement), e neppure appaiono meramente funzionali a un rientro nei ranghi dell'ortodossia o in vista di un edificante superamento per giungere a una meta più elevata, coincidente spesso con una sublimazione nel divino (alludendo, dunque, all'erranza sessuale quale tappa necessaria e provvisoria di un iter di formazione e perfezionamento).

L'amore saffico si propone piuttosto come un'alternativa - discussa e giustificata ampiamente, specie nella favola pastorale - a quello eterosessuale, di rado paritario. Un'alternativa che consente alla donna di riappropriarsi del proprio corpo e della propria identità con pienezza, abbracciando una castitas che non è privazione, bensì compiutezza: del sé, della propria natura altra rispetto agli stereotipi, dei propri aneliti intellettuali.

Una dimensione articolata e ricca questa che emerge dalle pagine della Nostra, e che - forse non a caso - soprattutto i suoi personaggi maschili sembrano o desiderano deliberatamente ignorare, svilire, contrastare. Nella realtà, possiamo immaginare che parimenti fu erto il cammino della vicentina

22 Per approfondire la tematiche menzionate, cfr. Giannetti (2009). 
"Meglio ancora delle sue opere, che nessuno più legge": note per la riscoperta di Maddalena

Campiglia (1553-1595)

Anna Lisa Somma

verso l'emancipazione letteraria, arduo il districarsi fra gender e genere, tra armature tematiche e stilistiche imposte in relazione all'appartenenza a un sesso, uno status o una ferrea griglia di comportamento. Eppure, a quanto ci è dato desumere dalle scarse notizie biografiche sul suo conto e dai suoi scritti, la donna non smise mai d'impiegare le sue risorse per tentare di infondere alla sua voce una tempra letteralmente sui generis; la stessa che - ci auguriamo possa, a distanza di cinque secoli, in virtù della sua grazia caparbia, far riemergere il nome di Maddalena Campiglia dall'oblio, tenendo così fede al motto da lei prescelto: TEMPORE SIC DURO. 
"Meglio ancora delle sue opere, che nessuno più legge": note per la riscoperta di Maddalena

Campiglia (1553-1595)

Anna Lisa Somma

\section{Riferimenti bibliografici}

BOSSIER, Philiep. Female writing and the use of literary byways. Pastoral drama by Maddalena Campiglia (1553-1595). In MONTOYA, Alicia; GILLEIR, Anke; VAN DIJK, Suzan (eds.). Women Writing Back / Writing Women Back. Transnational Perspectives from the Late Middle Ages to the Dawn of the Modern Era. Leiden: Brill, 2010, pp. 115-133.

ID. Non si è fermato il desiderio che nacque in me. Maddalena Campiglia e la ribellione alle convenzioni poetiche. In CORSARO, Antonio; HENDRIX, Harald; PROCACCIOLI, Paolo (eds.). Autorità, modelli e antimodelli nella cultura artistica e letteraria tra Riforma e Controriforma: atti del Seminario internazionale di studi, Urbino-Sassocorvaro, 9-11 novembre 2006. Manziana: Vecchiarelli, 2007, pp. 55-63.

CALDERARI, Cesare. Il trofeo della croce. Vicenza: Appresso Agostino della Noce, 1588.

CAMPIGLIA, Maddalena. Discorso sopra 1'Annonciatione della Beata Vergine, \& la Incarnatione del S.N. Giesu Christo. Vicenza: Appresso Perin libraro \& Giorgio Greco compagni, 1585.

EAD. Flori, a Pastoral Drama. Edited with Introduction and Notes by Virginia Cox and Lisa Sampson. Translated by Virginia Cox. Chicago London: The University of Chicago Press, 2004.

EAD. So che donna amo donna: la "Calisa" di Maddalena Campiglia. A cura di Carlachiara Perrone. Galatina: Congedo, 1996. 
"Meglio ancora delle sue opere, che nessuno più legge": note per la riscoperta di Maddalena

Campiglia (1553-1595)

Anna Lisa Somma

CHEMELLO, Adriana. Donne a poetar esperte. La "rimatrice dimessa" Maddalena Campiglia. Versants, 2003. P. 46, pp. 65-101.

COX, Virginia. Women's Writing in Italy, 1400-1650. United States of America: Johns Hopkins University Press, 2008, p. 158.

DUBY, Georges; PERROT, Michelle. Storia delle donne: dal Rinascimento all'età moderna. A cura di N. Zemon Davis e A. Farge. Roma - Bari: Editori Laterza, 2009.

GHERARDI, Sergio. Maddalena Campiglia, poetessa vicentina: nei testamenti del padre. Vicenza: La Serenissima, 2009.

GIANNETTI RUGGIERO, Laura. Lelia's Kiss. Imaging Gender, Sex, and Marriage in Italian Renaissance Comedy. Toronto - Buffalo - London: University of Toronto Press, 2009.

GRIMAL, Pierre. Enciclopedia dei miti. Milano: Garzanti editore, 1994. pp. 291-292.

JORDAN, Constance. Renaissance Feminism: Literary Texts and Political Models. Ithaca (NY): Cornell University Press, 1990.

MANTESE, Giovanni. Per un profilo storico della poetessa vicentina Maddalena Campiglia: Aggiunte e rettifiche. Archivio veneto, 1967. V serie, LXXXI, pp. 89-123.

MODERATA FONTE. Il merito delle donne. A cura di Adriana Chemello. Venezia-Mirano: Eidos, 1988. 
MORSOLIN, Bernardo. Maddalena Campiglia, poetessa vicentina del secolo XVI: episodio biografico. Vicenza: Paroni, 1882.

OVIDIO, Publio Nasone. I Fasti. Milano: Biblioteca universale Rizzoli, 1998.

ROSS, Sarah Gwyneth. The Birth of Feminism: Woman as Intellect in Renaissance Italy and England. Cambridge: Harvard University Press, 2009.

RUMOR, Sebastiano. Per una Poetessa del secolo XVI. Vicenza: Stabilimento Tipografico S. Giuseppe, 1897.

SARTORI, Diana. Maddalena Campiglia. In ARSLAN, Antonia; CHEMELLO, Adriana; PIZZAMIGLIO, Gilberto (eds.). Le stanze ritrovate: antologia di scrittrici venete dal Quattrocento al Novecento. Venezia: Eidos, 1994, pp. 57-68.

STUSSI, Alfredo. Scelte linguistiche e connotati regionali nella novella italiana. In ID. Lingua, dialetto e letteratura. Torino: Einaudi, 1993, p. 151.

TASSO, Torquato. Aminta. In ID. Poesie. A cura di Francesco Flora. Milano - Napoli: Riccardo Ricciardi Editore, 1952.

ID. Lettere di Torquato Tasso. In ID. Delle opere di Torquato Tasso con le controversie sopra la Gerusalemme liberata, e con le Annotazioni intere di varj Autori, notabilmente in questa impressione accresciute, vol. IX. Venezia: Appresso Steffano Monti e N. N. Compagno, 1738, lettera 506,318 . 
"Meglio ancora delle sue opere, che nessuno più legge": note per la riscoperta di Maddalena

Campiglia (1553-1595)

Anna Lisa Somma

ULTSCH (I), Lori J. Epithalamium Interruptum: Maddalena Campiglia's New Arcadia. MLN Italian Issue, CXX, 1, Jan. 2005, pp. 70-92.

EAD. (II). Maddalena Campiglia, Dimessa nel mondano cospetto?: Secular Celibacy, Devotional Communities, and Social Identity in Early Modern Vicenza. Forum Italicum: A Journal of Italian Studies, XXXIX, 2, September 2005, pp. 350-377.

ZARRI, Gabriella. Recinti: donne, clausura e matrimonio nella prima età moderna. Bologna: Il Mulino, 2000. 